\title{
VirtualToxLab ${ }^{T M}$ - In Silico Prediction of the Toxic (endocrine-disrupting) Potential of Drugs, Chemicals and Natural Products. Two Years and 2,000 Compounds of Experience: A Progress Report
}

\author{
Angelo Vedani, Martin Smiesko, Morena Spreafico, Ourania Peristera and Max Dobler \\ Biographics Laboratory 3R, CH-Basel and Department of Pharmaceutical Sciences, University of Basel, Switzerland
}

\begin{abstract}
Summary
The VirtualToxLab ${ }^{T M}$ is an in silico tool for predicting the toxic (endocrine-disrupting) potential of drugs, chemicals and natural products. It is based on a fully automated protocol and calculates the binding affinity of any molecule of interest towards a series of 12 proteins, known or suspected to trigger adverse effects and estimates the resulting toxic potential. In contrast to other approaches in the field, the technology allows to rationalize a prediction at the molecular level by interactively analyzing the binding mode of the tested compound with any target protein in $3 D$. The technology is accessible over the Internet (via a secure SSH protocol) and available for any science-oriented organization.

The toxic potential - a complex value derived from the individual binding affinities, their standard deviation and the quality of the underlying model (number and ratio of training and test compounds, activity range covered) - of existing and hypothetical compounds is estimated by simulating and quantifying their interactions towards a series of macromolecular targets at the molecular level using automated flexible docking combined with multi-dimensional QSAR (mQSAR). Currently, those targets comprise 12 proteins: the androgen, aryl hydrocarbon, estrogen $\alpha / \beta$, glucocorticoid, mineralocorticoid, thyroid $\alpha / \beta$ liver $X$ and the peroxisome proliferator-activated receptor $\gamma$ as well as the enzymes cytochrome P450 $3 A 4$ (CYP 3A4) and $2 A 13$ (CYP 2A13).

Up to date, the technology has been used to predict the toxic potential for more than 2,000 drugs, chemicals and natural compounds. All results are posted in the Internet - in this account, a few will be discussed in detail with reference to the molecular mechanisms triggering the adverse effect.
\end{abstract}

Keywords: VirtualTox $L a b^{T M}$, in silico prediction of the toxic (endocrine-disrupting) potential, toxicity mechanisms, reduction of animal testing

\section{Introduction}

Toxic agents, particularly those that exert their actions with a great deal of specificity, act frequently via receptors to which they bind with high affinity. This phenomenon is referred to as receptor-mediated toxicity. Examples of soluble intracellular receptors that are important in mediating toxic responses include the glucocorticoid receptor, which is involved in mediating toxicity associated effects such as apoptosis of lymphocytes as well as neuronal degeneration as a response to stress, the peroxisome proliferator-activated receptor, which is associated with hepa- tocarcinogenesis in rodents, and the aryl hydrocarbon receptor, which is involved in a whole range of toxic effects (see, for example, Gustaffson, 1995). Harmful effects of drugs and chemicals can often be associated with their binding to molecules other than their primary target - macromolecules involved in biosynthesis, signal transduction, transport, storage and metabolism (Rihova, 1998; Fischer 2000; Hestermann et al., 2000; Lukasiuk and Pitkänen, 2000; Rymer and Good, 2001; Hampson and Grimaldi, 2002; Oliver and Roberts, 2002).

Toxicity testing - as mandated by international regulations for drug development and chemical safety - is still associated 
with stressful animal tests. While many in vitro approaches have been devised to target the various aspects of toxicological phenomena, they require a chemical or drug molecule to be physically present (i.e. synthesized) before testing. In contrast hereto, computational approaches can be applied to hypothetical substances, as their 3D structure can be readily generated in silico. Nowadays computer power permits scanning larger batches of compounds in moderate time spans. Toxicity-modeling algorithms are typically based on quantitative structure-activity relationships, neuronal networks, artificial intelligence or rule-based expert systems. In previous accounts (Vedani at al., 2007a, 2005a), we provided an in-depth description of the underlying docking and QSAR technologies, their capabilities and limitations for modeling toxic phenomena. In this account, we discuss the first results: the toxic potential of more than 2,000 drugs, chemicals and natural products as determined in silico.

\section{Methods}

The philosophy underlying the VirtualToxLab ${ }^{\mathrm{TM}}$ is referred to as mixed-model QSAR. In a first step, the compound of interest is flexibly docked to the (human) target protein and all feasible poses (potential binding modes) are sampled into a 4D data set (software Yeti/AutoDock; Vedani et al., 2005b; Spreafico et al., 2009). In a next step, these data are used as input for multi- dimensional QSAR (mQSAR; software Quasar: Vedani et al., 2000a,b; Vedani and Dobler 2002; Vedani et al., 2005b, 2006, 2007a,b; Spreafico et al., 2009) for quantifying the binding affinities. Finally, the toxic potential is calculated from the individual binding affinities, their standard deviation and the quality of the underlying model (number and ratio of training and test compounds, activity range covered). Mixed-model QSAR was applied to the estrogen receptor $\alpha / \beta$ (Vedani et al., 2005b; Vedani et al., 2008), androgen receptor (Lill et al., 2005), the peroxisome proliferator-activated receptor $\gamma$ (Vedani et al., 2007b), the glucocorticoid receptor (Spreafico et al., 2009), the thyroid receptor $\alpha / \beta$ (Vedani et al., 2007a), the aryl hydrocarbon receptor (Vedani et al., 1999, 2007, 2008), the mineralocorticoid receptor (Peristera et al., in press) and the enzyme cytochrome P450 3A4 (Lill et al., 2006).

Yeti/AutoDock: Flexible docking aims at identifying all potential binding modes (orientations, conformations) of a small molecule within the binding pocket of a protein. The underlying protocol addresses two aspects of ligand-protein binding which would seem to be of utmost importance: 1. simulation of induced fit, i.e. allowing the protein to adapt its shape to the different orientations and conformations of the small molecule during the search procedure and 2. consideration of solvent effects (water). In our approach, the sampling is based on a Monte-Carlo/ Metropolis protocol. In addition, the implemented minimizer features a directional force field, which is particularly efficient

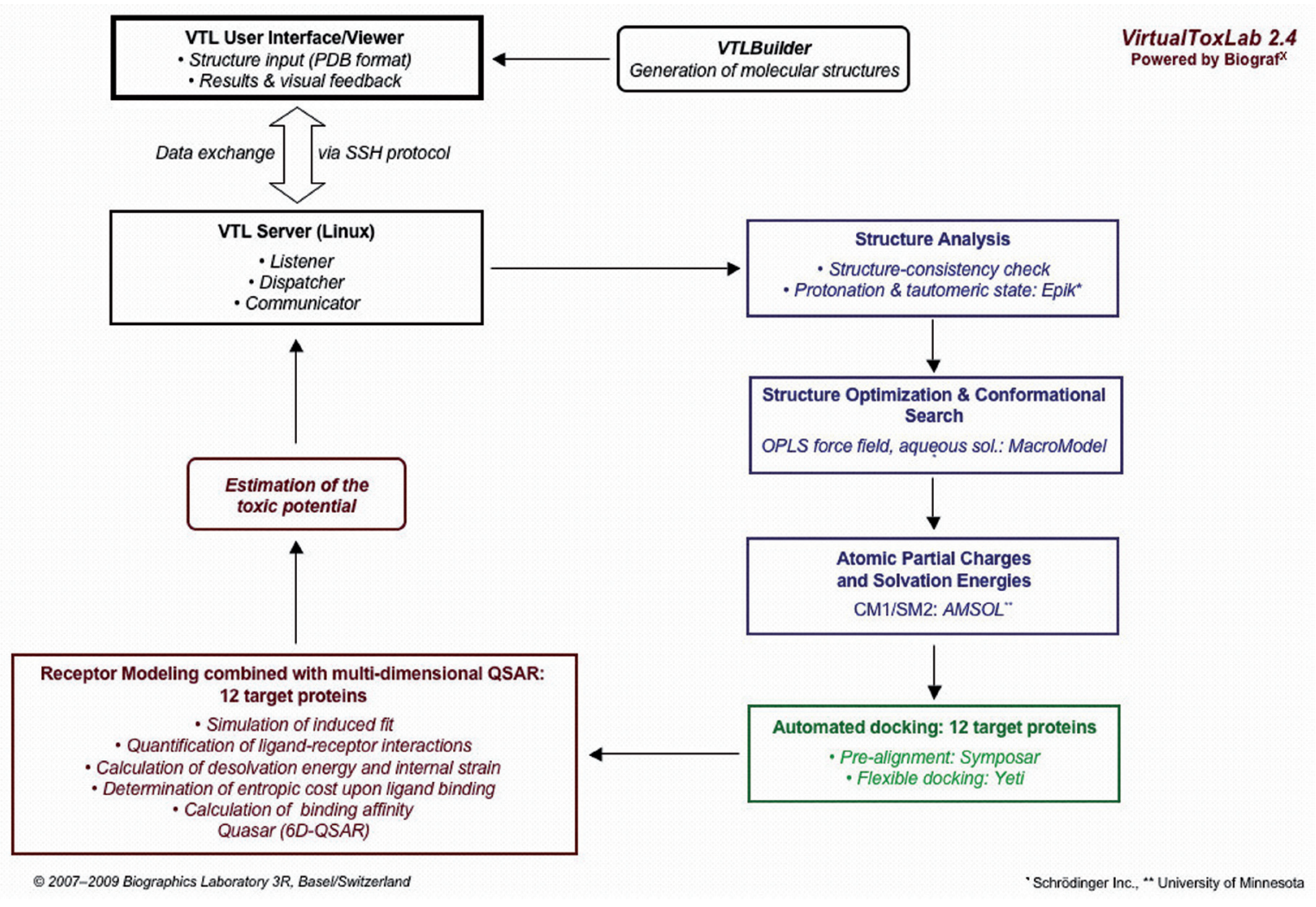

Fig. 1: Flow chart of the VirtualToxLab ${ }^{\text {TM }}$ 
in simulating hydrogen bonds and metal-ligand interactions (Vedani and Huhta 1990, 1991).

Quasar - a mQSAR tool developed at the Biographics Laboratory $3 R$ - allows to simultaneously consider different positions, orientations, conformations, tautomeric and protonation states of the ligand molecule (4D), different induced-fit scenarios (5D) and solvation models (6D). Quasar generates a family of quasi-atomistic receptor surrogates that are optimized by means of a genetic algorithm. The hypothetical receptor site is characterized by a three-dimensional surface that surrounds the ligand molecules at van der Waals distance and which is populated with atomistic properties mapped onto it. The topology of this surface mimics the three-dimensional shape of the binding site; the mapped properties represent other information of interest, such as hydrophobicity, electrostatic potential and hydrogen-bonding propensity (Vedani et al., 1998; Vedani and Dobler, 2002; Vedani et al., 2005b). For scoring the proteinligand interactions, Quasar employs the following function (equation 1):

$$
\begin{aligned}
& \begin{aligned}
E_{\text {binding }}= & E_{\text {ligand-receptor }}-E_{\text {ligand desolvation }}-E_{\text {ligand internal strain }} \\
& -T \Delta S-E_{\text {induced fit }} \\
\text { where } E_{\text {ligand-receptor }}= & E_{\text {electrostatic }}+E_{\text {van der Waals }} \\
& +E_{\text {hydrogen bonding }}+E_{\text {polarization }}
\end{aligned}
\end{aligned}
$$

$\Delta \mathrm{G}_{\text {binding }}=|\mathrm{a}| \cdot \mathrm{E}_{\text {binding }}+\mathrm{b}$
Using the ligands of the training set, a linear regression of the experimental $\Delta \mathrm{G}_{\text {binding }}$ and calculated $\mathrm{E}_{\text {binding }}$ is then obtained (equation 2). The coefficients $a$ and $b$ are derived from the correlation of the training set in cross-validation mode and, later on, applied to molecules of the test set or new compounds for which binding affinity should be predicted.

VirtualToxLab ${ }^{\mathrm{TM}}$ : It combines automated, flexible docking with mQSAR to simulate and quantify the binding of any compound of interest to the 12 currently implemented protein models (androgen, aryl hydrocarbon, estrogen $\alpha / \beta$, glucocorticoid, mineralocorticoid, thyroid $\alpha / \beta$ liver $X$, peroxisome proliferator-activated receptor $\alpha$; enzymes cytochrome P450 3A4 and 2A13) and to estimate the resulting toxic potential. The flow chart is depicted in Figure 1. The VirtualToxLab ${ }^{\mathrm{TM}}$ distribution includes a web interface with an integrated 3D viewer (Figure 2) and a 3D model builder (Figure 3 ) to generate the three-dimensional structures of the compounds to be tested. Results include the binding affinities towards the 12 target proteins along with the standard deviation and the 3D coordinates of the corresponding complexes - to be interactively viewed in real-time $3 \mathrm{D}$ or to be downloaded for further analyses or processing. All data (input and output) are transferred over a secure SSH protocol. After completion of a task, all data (except the log file and the 3D coordinates) are automatically and irreversibly deleted from the server. After downloading the pertinent data, the user can delete all remaining data. Complete details on the technology are given in http://www.biograf.ch/downloads/VirtualToxLab.pdf

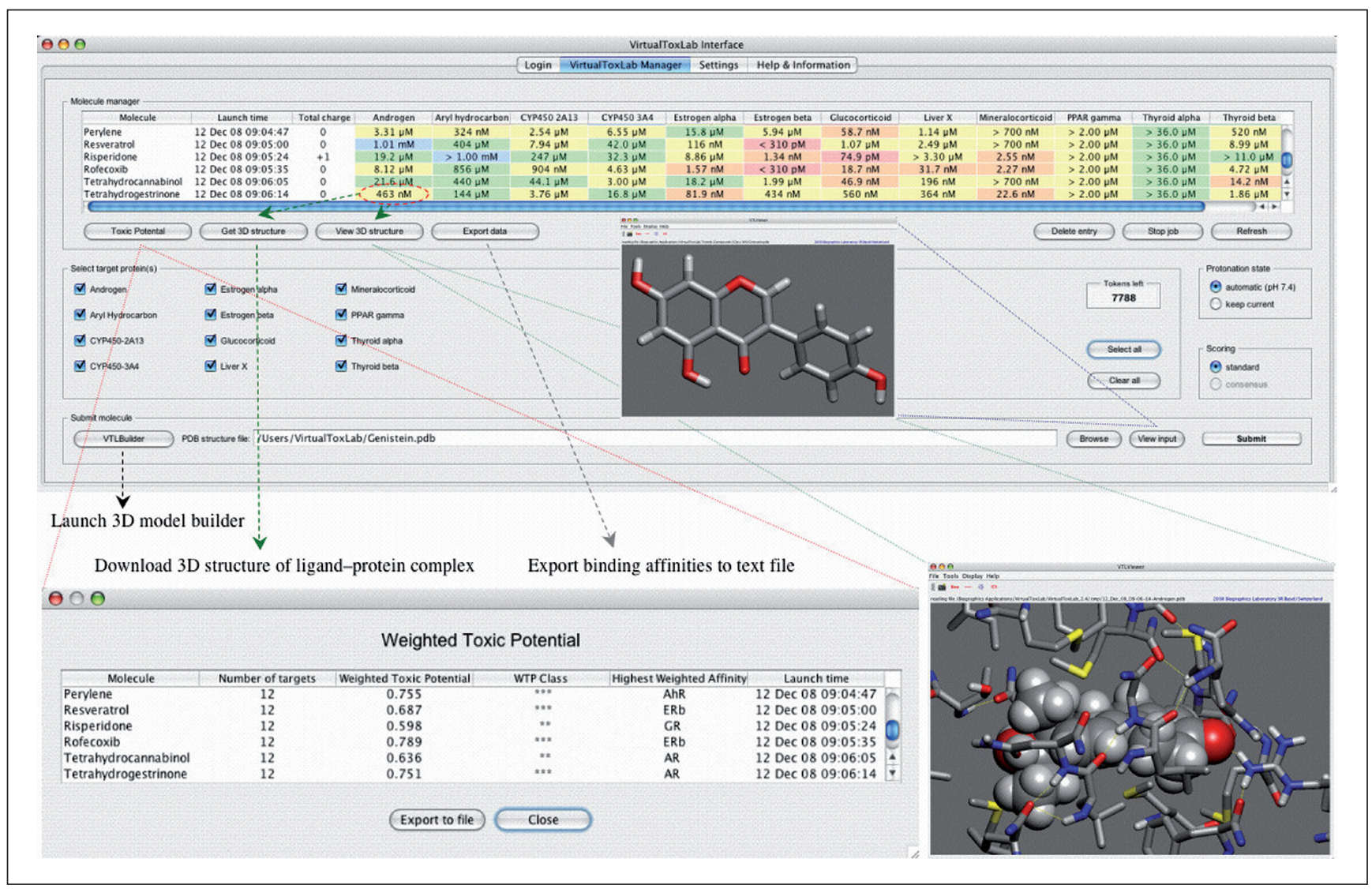

Fig. 2: VirtualToxLab ${ }^{\mathrm{TM}}$ : Web interface with integrated 3D viewer (the compound shown is coumestrol) 


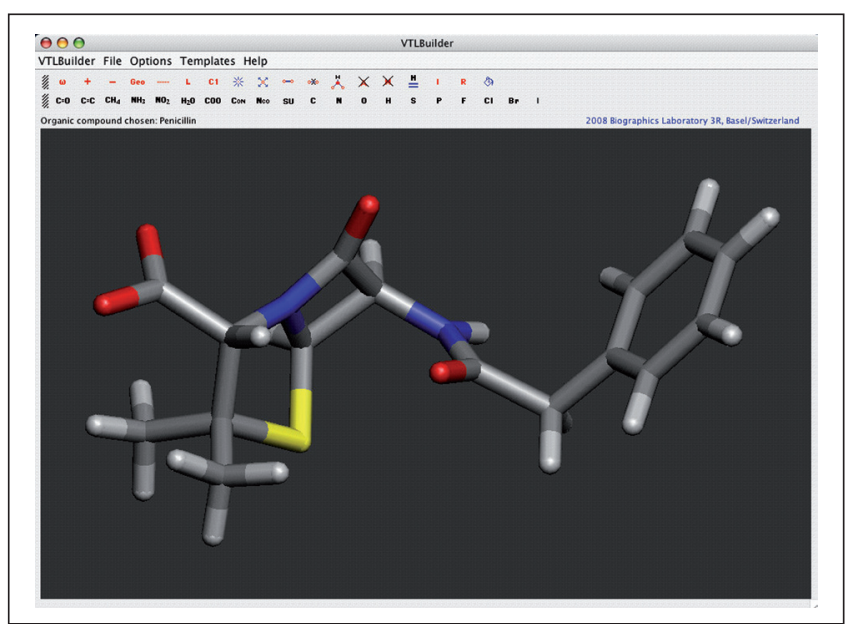

Fig. 3: VirtualToxLab ${ }^{\mathrm{TM}}$ : 3D model builder (the compound shown is benzylpenicillin)

Tables 1 and 2 give the details of the receptor models used in the VirtualToxLab ${ }^{\mathrm{TM}}$, in particular, the weight of the individual models towards the toxic potential. It depends on the number of employed compounds, the training-to-test set ratio and the activity range covered by the model. In order to compute the contributions of the individual binding affinities towards the toxic potential, the former are normalized according to equation 3 . affinity $<$ affinity model, lowest $\rightarrow$ affinity normalized $=0.0$

affinitymodel, lowest $<$ affinity $<$ affinitymodel, highest $\rightarrow$ affinitynormalized $=$ $\log ($ affinitymodel, lowest) $-\log$ (affinity) / log (affinitymodel, highest affinity model, highest)

affinity $>$ affinity model, highest $\rightarrow$ affinity normalized $=1.0$

Next, the weighted individual toxic potential, $\mathrm{wTP}_{\text {individual }}$, is calculated:

wTP $_{\text {individual }}=$ affinitynormalized $\times$ weight $_{\text {stand. }}$ dev.. weight $_{\text {target }}$

weight $_{\text {standard deviation }}=1.0-\sqrt{ } 0.1 \times$ (stand. dev. / affinity)

weight $_{\text {target }}=$ rating of the individual models (cf. Table 2 )

Therefrom, the overall toxic potential ( $\left.\mathrm{wTP}_{\text {overall }}\right)$ is determined as follows: First, the $12 \mathrm{wTP}_{\text {individual }}$ are ranked by their value. Then, their contribution to the $\mathrm{wTP}_{\text {overall }}$ is summed up according to equation 5 .

WTP $_{\text {overall }}=\sum\left(\begin{array}{c}1.0-\mathrm{wTP}_{\mathrm{n}=1} \\ \mathrm{wT}_{\text {overall, }} \text { current }\end{array}\right) \mathrm{x}$ WTP individual, $\mathrm{n}$

The $\mathrm{wTP}_{\text {overall }}$ is a degressive, non-linear function that ranges from 0.0 (benign) to 1.0 (extreme). As some of the rules for its derivation are quite arbitrary, the $\mathrm{WTP}_{\text {overall }}$ should not be overvalued but, instead, interpreted as a toxicity alert (cf. Figure 4).

Tab. 1: VirtualToxLab ${ }^{\text {TM}}$ : In silico validation of the underlying receptor models

\begin{tabular}{|c|c|c|c|c|c|c|c|c|}
\hline Systema & $\begin{array}{l}\text { training+test=total; } \\
\text { compound classes }\end{array}$ & $q^{2}$ & rms training & max. training & $p^{2}$ & rms test & max. test & $\mathrm{fn} / \mathrm{fp} \mathrm{p}^{\mathrm{b}}$ \\
\hline Androgen & $88+26=114 ;$ eight & 0.858 & 1.7 & 7.8 & 0.792 & 1.6 & 13.9 & $1 / 1$ \\
\hline Aryl hydrocarbon & $105+35=140 ;$ eight & 0.824 & 1.8 & 10.2 & 0.769 & 2.3 & 13.5 & $0 / 2$ \\
\hline Estrogen a & $80+26=106 ;$ six & 0.895 & 2.0 & 8.6 & 0.892 & 2.9 & 9.5 & $0 / 0$ \\
\hline Estrogen $\beta$ & $72+24=96 ;$ five & 0.802 & 2.0 & 8.8 & 0.699 & 2.7 & 13.4 & $0 / 2$ \\
\hline Glucocorticoid & $88+22=110 ;$ four & 0.702 & 1.5 & 9.9 & 0.719 & 1.6 & 4.9 & $0 / 0$ \\
\hline Liver $\mathrm{X}$ & $40+12=52 ;$ two & 0.763 & 1.2 & 7.5 & 0.697 & 1.3 & 3.3 & $0 / 0$ \\
\hline Mineralocorticoid & $40+8=48 ; t w o$ & 0.810 & 1.0 & 3.3 & 0.661 & 1.7 & 4.2 & $0 / 0$ \\
\hline PPARY & $75+20=95 ;$ two & 0.832 & 1.4 & 6.2 & 0.723 & 1.4 & 3.9 & $0 / 0$ \\
\hline Thyroid a & \multirow{2}{*}{ \} $64+18=82 ;$ four } & 0.919 & 1.8 & 4.3 & 0.814 & 2.5 & 10.0 & $0 / 1$ \\
\hline Thyroid $\beta$ & & 0.909 & 2.0 & 7.7 & 0.796 & 2.7 & 8.8 & $1 / 0$ \\
\hline CY2A13 & $18+6=24 ; \operatorname{six}$ & 0.854 & 0.8 & 2.0 & 0.661 & 0.8 & 1.7 & $0 / 0$ \\
\hline CYP3A4 & $38+10=48 ;$ eighteen & 0.825 & 2.7 & 7.0 & 0.659 & 3.8 & 7.1 & $0 / 0$ \\
\hline
\end{tabular}

$q^{2}=$ cross-validated $r^{2}, p^{2}=$ predictive $r^{2}$; the rms and maximal deviation from the experimental binding affinity is given as factor in $\mathrm{K}_{\mathrm{i}}$ or $\mathrm{IC}_{50}$. a Experimental data underlying the various models can be found in the primary references to the models or compiled for all systems under http://www.biograf.ch/index.php?id=projects\&subid=biodata.

b Because a correlation between experimental binding affinities and toxicological classes has only been established for few receptors (cf. Rannug et al., 1991), we have arbitrarily defined a factor of 10 off the experimental value as threshold. $\mathrm{fn}=$ false-negative, $\mathrm{fp}=\mathrm{false}-\mathrm{positive}$ compounds.

References: AR (Lill et al., 2005); AhR (Vedani et al., 2008), ERa (Vedani et al., 2005b), ERß (Vedani et al., 2008); GR (Spreafico et al., 2009); LXR (in preparation); MR (Peristera et al., in press); PPARy (Vedani et al., 2007b); TRaß (Vedani et al., 2007a); 2A13 (Vedani et al., 2008); 3A4 (Lill et al., 2006). 
Tab. 2: VirtualTox Lab ${ }^{T M}$ : overall rating of the underlying receptor models

\begin{tabular}{llllll}
\hline System & \multicolumn{1}{l}{ compounds } & activity range & protein & $\mathrm{K}_{\mathrm{i} \text { or IC50 }}$ & rating ${ }^{\mathrm{a}}$ \\
\hline Androgen & $88+26=114$ & $46 \mathrm{mM}-1.7 \mathrm{nM}$ & human & rat & 0.786 \\
Aryl hydrocarbon & $105+35=140$ & $1.0 \mathrm{mM}-3.2 \mathrm{nM}$ & human b & rat & 0.806 \\
Estrogen a & $80+26=106$ & $2.8 \mathrm{mM}-0.23 \mathrm{nM}$ & human & human & 0.957 \\
Estrogen $\beta$ & $72+24=96$ & $69 \mu \mathrm{M}-0.31 \mathrm{nM}$ & human & human & 0.724 \\
Glucocorticoid & $88+22=110$ & $2.5 \mu \mathrm{M}-0.05 \mathrm{nM}$ & human & human & 0.663 \\
Liver X & $40+12=52$ & $3.3 \mu \mathrm{M}-0.22 \mathrm{nM}$ & human & human & 0.474 \\
Mineralocorticoid & $40+8=48$ & $0.70 \mu \mathrm{M}-0.60 \mathrm{nM}$ & human & human & 0.295 \\
PPARY & $75+20=95$ & $2.0 \mu \mathrm{M}-0.69 \mathrm{nM}$ & human & human & 0.513 \\
Thyroid a & $64+18=82$ & $36 \mu \mathrm{M}-0.09 \mathrm{nM}$ & human & human & 0.663 \\
Thyroid $\beta$ & $64+18=82$ & $11 \mu \mathrm{M}-0.68 \mathrm{nM}$ & human & human & 0.550 \\
CYP 2A13 & $18+6=24$ & $0.43 \mathrm{mM}-0.46 \mu \mathrm{M}$ & human & human & 0.178 \\
CYP 3A4 & $38+10=48$ & $2.0 \mathrm{mM}-9.0 \mathrm{nM}$ & human & human & 0.423 \\
\hline
\end{tabular}

a The rating (ranging from 0.0 to 1.0) depends on the number of employed ligands, the training:test set ratio and the covered affinity range. This factor is used for weighting the toxic potential.

$\mathrm{b}$ based on a homology model; for all other proteins, the 3D structure has been experimentally determined and is available through the Protein Data Bank (http://www.rcsb.org/pdb)

\section{Results and discussion}

Using the VirtualToxLab ${ }^{\text {TM }}$ technology, we have estimated the toxic (endocrine-disrupting) potential of more than 2,000 compounds - drugs, chemicals and natural products. Full details are given at http://www.virtualtoxlab.org. Two compound classes were analyzed in depth: psychotropic drugs (Spreafico et al., 2009) and anabolic steroids (Peristera et al., in press). Here, we shall present four representative cases: dibenzo[ah]anthracene, tetrahydrogestrinone, resveratrol and diethylstilbestrol; the calculated toxic potential of 78 other compounds is given in Figure 4 and Table 7, respectively.

Dibenzo[ah]anthracene is a known carcinogen (Brunström et al., 1991). Its toxic potential in silico is estimated as 0.864 , which is classified as extreme. The individual binding affinities towards the 12 target proteins tested in the VirtualToxLab ${ }^{\mathrm{TM}}$ are given in Table 3. In particular, dibenzo[ah]anthracene is computed to bind with $5.8 \mathrm{nM}$ to the aryl hydrocarbon receptor (experimental affinity = $16 \mathrm{nM}$; cf. Mason et al., 1986). This can be well understood when inspecting the binding mode of dibenzo[ah]anthracene at the aryl hydrocarbon receptor (Figure 5). The polyaromatic hydrocarbon is perfectly accommodated in the predominantly hydrophobic pocket lined by residues Phe4, Phe12, Phe68, Met65, His8 and His43. The negative desolvation energy and the rigidity (entropic terms) of dibenzo[ah] anthracene contribute further to the high binding affinity. Substantial affinities are also computed towards the GR $(58 \mathrm{nM})$ and the LXR (74 $\mathrm{nM})$.

Tetrahydrogestrinone (also known as "The Clear") is a synthetic anabolic steroid that primarily binds to the androgen receptor (Death et al., 2004) and was banned by the FDA in 2003. Our results (Table 4) are in agreement with these findings, i.e. the calculated binding affinity towards the androgen receptor $(16 \mathrm{nM})$ is significantly stronger than that towards the estrogen receptor $(\alpha: 310 \mathrm{nM} ; \beta: 120 \mathrm{nM})$. A similarly high affinity is computed towards the mineralocorticoid receptor $(28 \mathrm{nM})$. At the androgen receptor, the high affinity can be rationalized at the molecular level through the three hydrogen bonds with Asn37, Arg84 and Thr209, respectively, tetrahydrogestrinone engages in (Figure 6). The computed toxic potential is 0.814 , classifying the compound as quite dangerous.

Diethylstilbestrol is a non-steroidal anti-inflammatory drug that has been withdrawn in 1971 due to its teratogenic effects (Mittendorf, 1995). In our simulation, high affinities towards the estrogen receptor $\alpha(57 \mathrm{nM})$ and $\beta(14 \mathrm{nM})$ are computed (Table $5)$; the computed toxic potential is 0.721 , classifying the compound as quite dangerous with respect to endocrine disruption. At the estrogen receptor $\alpha$, the high affinity can be explained by the strong hydrogen bonds to Glu353, Arg394 and His524, respectively, and the interaction of the two aromatic rings and the ethyl groups with the hydrophobic part of the binding pocket (Figure 7).

Resveratrol is a phytoalexin produced naturally by several plants when under attack by pathogens such as bacteria or fungi. Resveratrol has also been produced by chemical synthesis and is sold as a nutritional supplement. It extends the life span of several short-living species of animals; however, this effect has not yet been demonstrated in mammals. In mouse and rat, resveratrol has anti-cancer, anti-inflammatory, bloodsugar lowering and other beneficial cardiovascular effects (see, for example, Vingtdeux et al., 2008; Fan et al., 2008; Markus and Morris, 2008; Rocha-Gonzales et al., 2008). Resveratrol is found in the skin of red grapes and in red wine. In our simulation, significant affinities towards the estrogen 


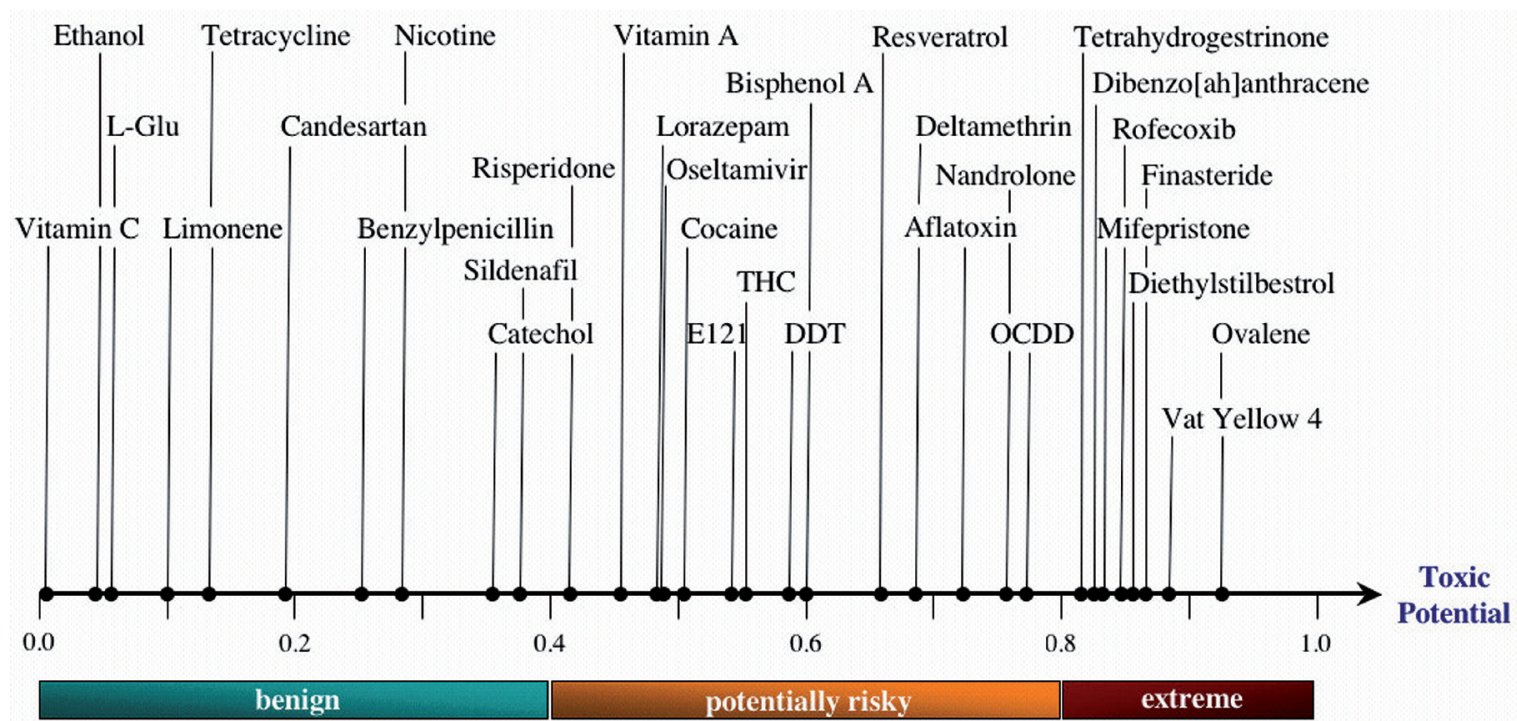

Fig. 4: Toxicity alerts as generated by the VirtualToxLab ${ }^{\mathrm{TM}}$

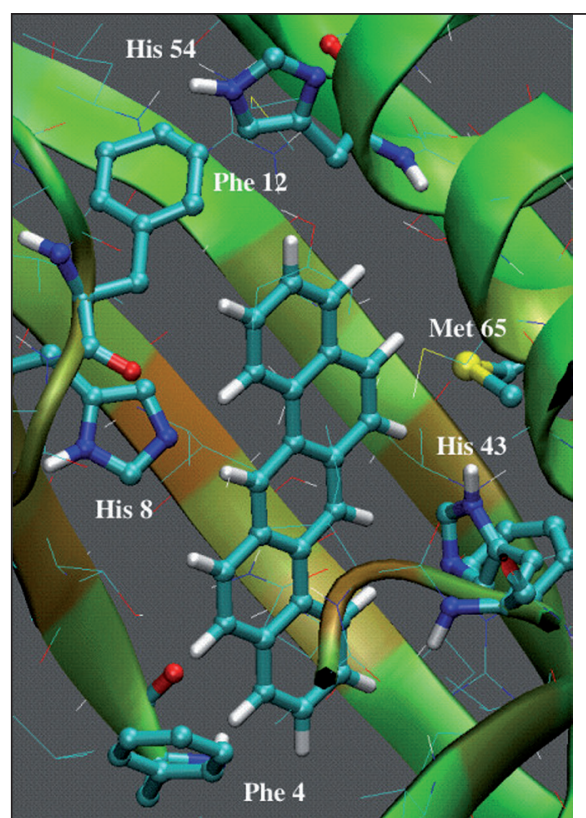

Fig. 5: Details of the binding of dibenzo[ah]anthracene to the aryl hydrocarbon receptor (3D image)

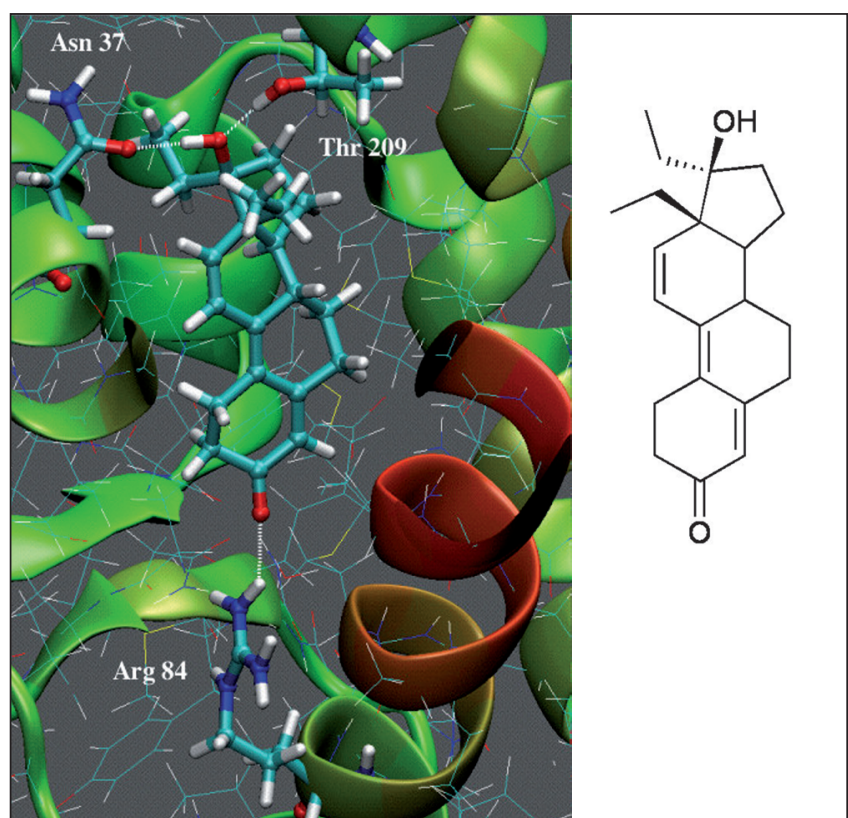

Fig. 6: Details of the binding of tetrahydrogestrinone to the androgen receptor (3D image)

Tab. 3: VirtualToxLab ${ }^{T M}$ : Calculated binding affinities of dibenzo[ah]anthracene

\begin{tabular}{|l|l|l|l|l|l|l|l|l|l|l|l|}
\hline AR & AhR & $2 \mathrm{~A} 13$ & $3 \mathrm{~A} 4$ & ERa & ER $\beta$ & GR & LXR & MR & PPARY & TRa & TR $\beta$ \\
\hline $3.5 \mu \mathrm{M}$ & $5.8 \mathrm{nM}$ & $1.4 \mu \mathrm{M}$ & $1.7 \mu \mathrm{M}$ & $1.3 \mu \mathrm{M}$ & $430 \mathrm{nM}$ & $58 \mathrm{nM}$ & $74 \mathrm{nM}$ & $590 \mathrm{nM}$ & $7.8 \mu \mathrm{M}$ & $4.2 \mathrm{mM}$ & $260 \mathrm{nM}$ \\
\hline
\end{tabular}

Tab. 4: VirtualToxLab ${ }^{T M}$ : Calculated binding affinities of tetrahydrogestrinone

\begin{tabular}{|l|l|l|l|l|l|l|l|l|l|l|l|}
\hline AR & AhR & $2 \mathrm{~A} 13$ & $3 \mathrm{~A} 4$ & ERa & ER & GR & LXR & MR & PPARY & TRa & TR \\
\hline $16 \mathrm{nM}$ & $25 \mu \mathrm{M}$ & $62 \mu \mathrm{M}$ & $14 \mu \mathrm{M}$ & $310 \mathrm{nM}$ & $120 \mathrm{nM}$ & $210 \mathrm{nM}$ & $230 \mathrm{nM}$ & $28 \mathrm{nM}$ & $670 \mu \mathrm{M}$ & $>1.0 \mathrm{M}$ & $3.4 \mu \mathrm{M}$ \\
\hline
\end{tabular}




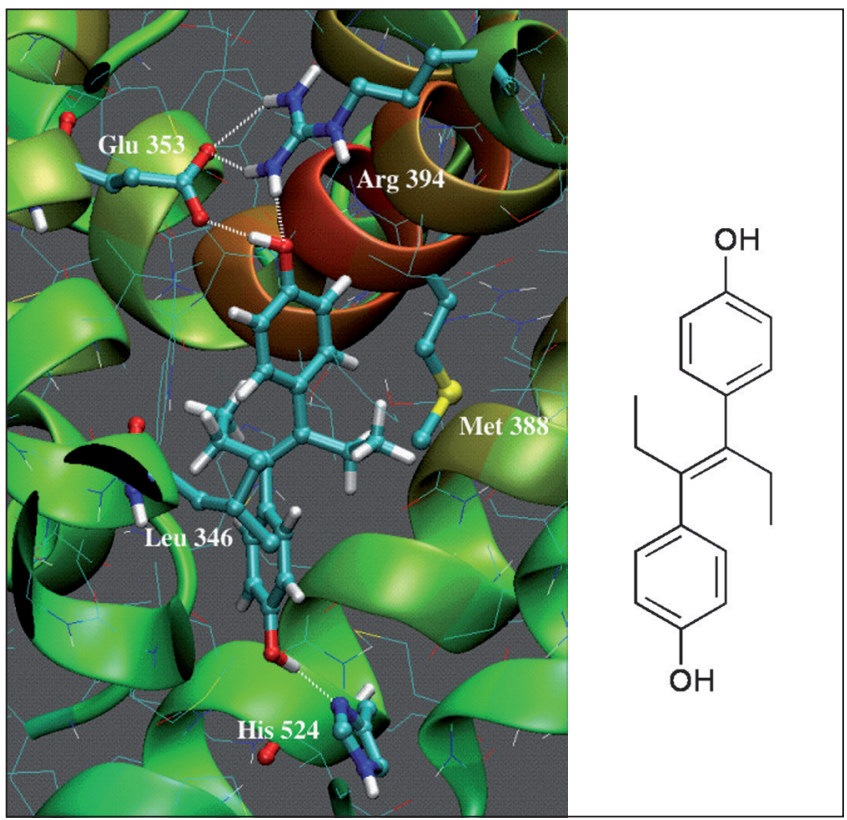

Fig. 7: Details of the binding of diethylstilbestrol to the estrogen receptor a (3D image)

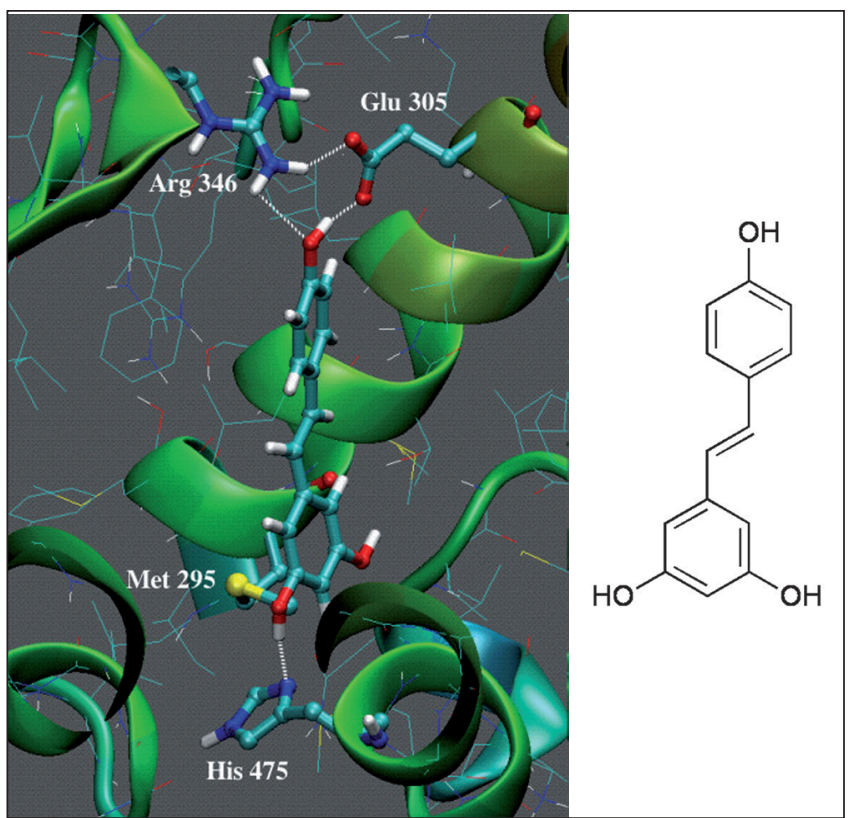

Fig. 8: Details of the binding of resveratrol to the estrogen receptor $\beta$ (3D image)

Tab. 5: VirtualToxLab ${ }^{T M}$ : Calculated binding affinities of diethylstilbestrol

\begin{tabular}{|c|c|c|c|c|c|c|c|c|c|c|c|}
\hline AR & AhR & $2 \mathrm{~A} 13$ & $3 A 4$ & ERa & $E R \beta$ & GR & LXR & MR & PPARY & TRa & TR $\beta$ \\
\hline $39 \mu \mathrm{M}$ & $450 \mu \mathrm{M}$ & $40 \mu \mathrm{M}$ & $30 \mu \mathrm{M}$ & $57 \mathrm{nM}$ & $14 \mathrm{nM}$ & $230 \mathrm{nM}$ & $10 \mu \mathrm{M}$ & $950 \mathrm{nM}$ & $1.4 \mathrm{mM}$ & $>1.0 \mathrm{M}$ & $60 \mu \mathrm{M}$ \\
\hline
\end{tabular}

Tab. 6: VirtualToxLab ${ }^{T M}$ : Calculated binding affinities of resveratrol

\begin{tabular}{|c|c|c|c|c|c|c|c|c|c|c|c|}
\hline AR & AhR & $2 \mathrm{~A} 13$ & $3 A 4$ & ERa & $E R \beta$ & GR & LXR & MR & PPARY & TRa & $\operatorname{TR} \beta$ \\
\hline $340 \mu \mathrm{M}$ & $5.1 \mathrm{mM}$ & $8.4 \mu \mathrm{M}$ & $11 \mu \mathrm{M}$ & $1.0 \mu \mathrm{M}$ & $2.3 \mathrm{nM}$ & $80 \mathrm{nM}$ & $5.1 \mu \mathrm{M}$ & $1.2 \mu \mathrm{M}$ & $130 \mu \mathrm{M}$ & $>1.0 \mathrm{M}$ & $6.7 \mu \mathrm{M}$ \\
\hline
\end{tabular}

receptor $\beta(2.3 \mathrm{nM})$ and the glucocorticoid receptor $(80 \mathrm{nM})$ are computed (Table 7); the computed toxic potential is 0.662 , classifying the compound as slightly dangerous with respect to endocrine disruption. At the estrogen receptor $\beta$, the high affinity can be explained by the strong hydrogen bonds to Glu305, Arg346 and His475, respectively (Figure 8). In contrast to diethylstilbestrol (cf. above), resveratrol has a clearly lower endocrine-disrupting potential. This is primarily due to the additional hydroxyl groups which i) are not positioned optimally for strong hydrogen bonding and ii) leads to a higher desolvation energy. In addition, it does not fit snugly to the binding pocket as it lacks the two ethyl groups (cf. Figures 7 and 8). To confirm this result further, we have performed molecular-dynamical simulations with both diethylstilbestrol and resveratrol. They indicate that resveratrol binds even more weakly - in contrast to diethylstilbestrol - as the key hydrogen bonds are not formed during the entire simulation $\left(1.0 \times 10^{-12} \mathrm{sec}\right.$ at $\left.25^{\circ} \mathrm{C}\right)$.
Table 7 lists the calculated toxic potential for 50 selected compounds. The complete listing for over 2,000 compounds (approx. 1,500 drugs, 500 chemicals, 150 natural compounds) is posted at http://www.biograf.ch/data/projects/virtualtoxlab_results.php.

False-negative predictions: compounds that do not exert their toxic or adverse effects via binding to one of the proteins currently tested in the VirtualToxLab ${ }^{\mathrm{TM}}$ are likely to go unnoticed. Examples include Ochratoxin A (a highly toxic mycotoxin; wTP $=0.405)$, coumarin (a plant toxin; $\mathrm{wTP}=0.384$ ) or ibotenic acid (a neurotoxin occurring in mushrooms; wTP $=0.085)$. The binding mode of very small compounds (MW $<100$ ) may not be correctly identified by the implemented protocol, which includes the sampling of up to 25 different poses, the eight energetically most favorable of which are used for the quantification in mQSAR. Such compounds include many of the chemicals used in the cosmetics industry. Here, semiautomated protocols, allowing for an exhaustive sampling, 
Tab. 7: VirtualToxLab ${ }^{T M}$ : Calculated endocrine-disrupting potential for 50 selected compounds

\begin{tabular}{|c|c|c|c|c|}
\hline Compound & calculated toxic potential & class & main target & drug or chemical class \\
\hline Aflatoxin & 0.732 & $* * *$ & ER $\beta$ & mycotoxin \\
\hline Amlodipine & 0.629 & $* *$ & GR & calcium channel blocker \\
\hline Auxin & 0.202 & & & phytohormone \\
\hline Benzo[a]anthracene & 0.734 & $* * *$ & AhR & polyaromatic hydrocarbon \\
\hline Benzo[a]pyrene & 0.810 & $* \star \star$ & AhR & polyaromatic hydrocarbon \\
\hline Benzylidene camphor & 0.637 & $\star *$ & $E R \beta$ & UV filter \\
\hline Benzylpenicillin & 0.254 & & & antibiotic \\
\hline Bisphenol A & 0.600 & ** & ER $\beta$ & polymer additive \\
\hline Ciprofloxacin & 0.311 & & & antibiotic \\
\hline Clofentezine & 0.587 & ** & AR & pesticide \\
\hline Clopiodogrel & 0.336 & * & GR & anti-platelet agent \\
\hline Cyclamate & 0.133 & & & artificial sweetener \\
\hline Dibenzo[ah]anthracene & 0.862 & 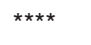 & AhR & polyaromatic hydrocarbon \\
\hline Diethylstilbestrol & 0.721 & $* \star \star$ & ERa & withdrawn drug \\
\hline Dimoxystrobin & 0.533 & $* *$ & GR & pesticide \\
\hline E100 & 0.606 & $* \star \star$ & GR & food dye \\
\hline E104 & 0.487 & * & GR & food dye \\
\hline E121 & 0.545 & ** & $\mathrm{AR}$ & food dye \\
\hline Ecstasy & 0.514 & ** & $\mathrm{AR}$ & designer drug \\
\hline Finasterid & 0.864 & 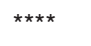 & ERa & antiandrogen \\
\hline Flanzinam & 0.733 & 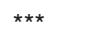 & AR & pesticide \\
\hline Fluticasone & 0.796 & 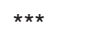 & ERa & corticosteroid \\
\hline Genistein & 0.674 & $\star *$ & ER $\beta$ & antioxidant \\
\hline Hexabromodiphenylether & 0.699 & $* \star \star$ & AR & flame retardant \\
\hline Isoxaflutole & 0.623 & $\star *$ & ERa & pesticide \\
\hline Limonene & 0.100 & & & flavorant \\
\hline Loracarbef & 0.422 & * & $E R \beta$ & antibiotic \\
\hline Lorazepam & 0.479 & ** & $\mathrm{AR}$ & psychotropic drug \\
\hline LSD & 0.568 & ** & GR & withdrawn drug \\
\hline Methylbenzylidene camphor & 0.656 & ** & $\mathrm{AR}$ & UV filter \\
\hline Methylparabene & 0.408 & * & $E R \beta$ & fungicide \\
\hline Mifepristone & 0.832 & 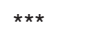 & $\mathrm{AR}$ & abortificant \\
\hline Nicotine & 0.386 & & & tobacco alkaloid \\
\hline Norethandrolone & 0.786 & 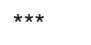 & AR & anabolic steroid \\
\hline Norethynodrel & 0.779 & 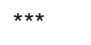 & AR & oral contraceptive \\
\hline Oseltamivir & 0.486 & * & GR & antiviral drug \\
\hline Paracetamol & 0.500 & $\star *$ & $E R \beta$ & analgesic drug \\
\hline Rofecoxib & 0.763 & $* * *$ & $\mathrm{AR}$ & withdrawn drug \\
\hline TCDD & 0.675 & $* * *$ & AhR & "Seveso dioxin" \\
\hline Tetracyclin & 0.234 & & & antibiotic \\
\hline THC & 0.553 & $\star \star *$ & AR & recreational drug \\
\hline Tetrahydrogestrinone & 0.814 & $* * *$ & $A R$ & anabolic steroid \\
\hline Thaliodomide & 0.513 & $* *$ & $A R$ & withdrawn drug \\
\hline Trimipramine & 0.562 & ** & GR & psychotropic drug \\
\hline Troglitazone & 0.707 & 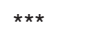 & AR & withdrawn drug \\
\hline Vitamin A & 0.454 & * & $\mathrm{AR}$ & retinoid \\
\hline Vitamin B2 & 0.478 & * & $E R \beta$ & riboflavin \\
\hline Vitamin C & 0.021 & & & ascorbic acid \\
\hline Vitamin D2 & 0.626 & ** & ER $\beta$ & ergocalciferol \\
\hline Warfarin & 0.314 & & & anticoagulant \\
\hline
\end{tabular}


would seem to be more appropriate. Finally, large compounds $(\mathrm{MW}>500)$ may trigger a more substantial induced fit that can presently be simulated.

False-positive predictions: as the underlying protocol quantifies exclusively thermodynamic aspects of ligand binding but ignores pharmacokinetic effects (adsorption, distribution, metabolism, excretion), a compound may not be present in significant amounts at the target protein and, hence, will not act as an agonist or antagonist (inhibitor or substrate). In the current version, metabolic products are not generated automatically but may be explicitly submitted by the user.

Consequently, the VirtualToxLab ${ }^{\text {TM }}$ should be employed to generate toxic alerts and, depending on the outcome, suggest further experiments to confirm or refute the findings. In the drug-discovery process, however, where larger series of hypothetical compounds must be screened, the technology should be extremely beneficial.

\section{Conclusions}

The VirtualToxLab ${ }^{\text {TM }}$ - an in silico technology developed at the Biographics Laboratory 3R - allows predicting the toxic (endocrine-disrupting) potential of drugs, chemicals and natural products. It is based on an automated protocol and calculates the binding affinity of any molecule of interest towards a series of 12 proteins, known or suspected to trigger adverse effects, and estimates the resulting toxic potential. Its input can easily be generated using the VTLBuilder (a 3D model builder) supplied with the distribution. The underlying interface employs a secure protocol (SSH) and allows up/download of all necessary data (compounds, 3D models, affinities, toxic potential). The calculation of the toxic potential of a medium-size compound requires 8-12 hours on our high-end computers. Currently, four computers featuring 32 processors are dedicated to the task.

The VirtualToxLab ${ }^{\mathrm{TM}}$ is available at a modest fee (cf. http:// www.virtualtoxlab.org) for science-oriented organizations. The fee is aimed at covering the effective costs associated with its operation (hardware infrastructure, support, third-party software licenses).

The associated model depository is continuously expanded. Currently, models for CYP450 2D6, CYP450 2C9, hERG and the pregnan-X receptor are under development.

\section{3R relevance}

The VirtualToxLab can contribute to a significant reduction in animal testing, as it allows for an early - i.e. before chemical synthesis - recognition of potentially harmful compounds. By removing those candidate substances from the evaluation pipeline, they will not be forwarded to any in vivo toxicity tests. Another field of application is testing chemicals for toxicity for example the 30,000 compounds that have to be retested by 2012 as defined in the European Commission's well-documented White paper on the strategy for a future chemicals policy
(2003) - and causing an estimated toll of 10 million laboratory animals. Here, our system could prove to be a useful in silico screening tool, as new compounds can be tested with only moderate human efforts. The importance of QSARs has more recently been acknowledged by the OECD (2003) and the Danish Environmental Protection Agency has taken the lead in the use of structure-based methods to prioritize hazardous chemicals (Cronin, 2003).

\section{References}

Brunström, B., Broman, D. and Näf, C. (1991). Toxicity and EROD-inducing potency of 24 polycyclic aromatic hydrocarbons (PAHs) in chick embryos. Arch. Toxicol. 65, 485489.

Cronin, M. (2003). A toxic gamble. Chemistry and Industry, 4, 13-14.

Death,A. K., McGrath, K. C., Kazlauskas, R. and Handelsmann, D. J. (2004). Tetrahydrogestrinone is a potent androgen and progestin. J. Clin. Endocrinol. Metab. 89, 2494- 2500.

Fan, E., Zhang, L., Jiang, S. and Bai, Y. (2008). Beneficial effects of resveratrol on arteriosclerosis. J. Med. Food 11, 610-614.

Fischer, B. (2000). Receptor-mediated effects of chlorinated hydrocarbons. Andrologia 32, 279-283.

Gustaffson, J.A. (1995). Receptor-mediated toxicity. Toxicol. Lett. 135, 465-470.

Hampson, A. J. and Grimaldi, M. (2002). 12-hydroxyeicosatetrenoate (12-HETE) attenuates AMPA receptor-mediated neurotoxicity: Evidence for a G-protein coupled HETE receptor. J. Neurosci. 22, 257-264.

Hestermann, E. V., Stegemann, J. J. and Hahn, M. E. (2000). Relative contribution of affinity and intrinsic efficacy to aryl hydrocarbon receptor ligand potency. Toxicol. Appl. Pharmacol. 168, 160-172.

Lill, M. A., Winiger, F., Vedani, A. and Ernst, B. (2005). Impact of induced fit on the ligand binding to the androgen receptor: A multidimensional QSAR study to predict endocrinedisrupting effects of environmental chemicals. J. Med.Chem. 48, 5666-5674.

Lill, M. A., Vedani, A., Dobler, M. (2004). Raptor - combining dual-shell representation, induced-fit simulation and hydrophobicity scoring in receptor modeling: Application towards the simulation of structurally diverse ligand sets. J. Med. Chem. 47, 6174-6186.

Lukasink, K. and Pitkanen, A. (2000). GABA(A)-mediated toxicity of hippocampal neurons in vitro. J. Neurochem. 74, 2445-2454.

Mason, G., Sawyer, T., Keys, et al. (1985). Polychlorinated dibenzofurans (PCDFs): Correlation between in vivo and in vitro structure-activity relationships. Toxicology 37, 1-12.

Markus, M. A. and Morris, B. J. (2008). Resveratrol in prevention and treatment of common clinical conditions of aging. Clin. Interv. Aging 3, 331-339.

Mittendorf, R. (1995). Teratogen update: carcinogenesis and teratogenesis associated with exposure to diethylstilbestrol (DES) in utero. Teratology 51, 435-445. 
OECD, 2003. http://webdomino1.oecd .org/comnet/env/models. nsf

Oliver, J. D. and Roberts, R. A. (2002). Receptor-mediated hepatocarcinogenesis - role of hepatocyte proliferation and apoptosis. Pharmacol. Toxicol.91, 1-7.

Peristera, O., Spreafico, M., Smisko, M. et al. (in press). Mixedmodel QSAR at the human mineralocorticoid receptor: Prediciting binding mode and affinity of anabolic steroids. Toxicol. Lett.

Rannug, U., Sjörgren, M., Rannug, A. et al. (1991). Use of artificial intelligence in structure-affinity correlations of 2,3,7,8tetrachloro-dibenzo-p-dioxin (TCDD) receptor ligands. Carcinogenesis 12, 2007-2015.

Rihova, B. (1998). Receptor-mediated targeted drug or toxin delivery. Adv. Drug Deliv. Rev. 29, 273-289.

Rocha-Gonzales, H. I., Ambriz-Tututti, M. and Granados-Soto, V. (2008). Resveratrol: a natural compound with pharmacological potential in neurodegenerative diseases. CNS Neurosci. Ther. 14, 234-247.

Rymer, D. L. and Good, T. A. (2001). The role of protein activation in the toxicity of amyloidogenic $A \beta(1-40), A \beta(25-35)$, and bovine calcitonin. J. Biol. Chem. 276, 2523-2530.

Spreafico, M., Smiesko, M., Lill, M. A. et al. (2009). Mixedmodel QSAR at the glucocorticoid receptor: Predicting the binding mode and affinity of psychotropic drugs. ChemMedChem 4, 100-109.

US Government (1996a): Safe Drinking Water Act Amendment. Public Law 104-182 (Section 136), http://www.epa.gov.safewater/sdwa/index.html

US Government (1996b): Food Quality Protection Act. Public Law 104-170 (Section 408), http://www.fda.gov/opacom/ laws/foodqual/fqpatoc.htm

Vedani, A., Spreafico, M., Peristera, O. and Smiesko, M. (2008). VirtualToxLab - In silico prediction of the endocrine-disrupting potential of drugs and chemicals. Chimia 5, 322-328.

Vedani, A., Zumstein, M., Lill, M. A. and Ernst, B. (2007a). Simulating $\alpha / \beta$ specificity at the thyroid receptor: Consensus scoring in multidimensional QSAR. ChemMedChem 2, 78-87.

Vedani, A., Decloux, A. V., Spreafico, M. and Ernst, B. (2007b). Predicting the toxic potential of drugs and chemicals in silico: A model for the peroxisome proliferator-activated receptor $\gamma$. Toxicol.Lett. 173, 17-23.
Vedani, A., Dobler, M. and Lill, M. A. (2006). The challenge of predicting drug toxicity in silico. Pharmacol. Toxicol. 99, 195-208.

Vedani, A., Dobler, M. and Lill, M. A. (2005a). Virtual test kits for predicting harmful effects triggered by drugs and chemicals mediated by specific proteins. ALTEX 22, 123- 134.

Vedani, A., Dobler, M. and Lill, M. A. (2005b). Combining protein modeling and 6D-QSAR - Simulating the binding of structurally diverse ligands to the estrogen receptor. J. Med. Chem. 48, 3700-3703.

Vedani, A. and Dobler, M. (2002). 5D-QSAR: The key for simulating induced fit? J. Med. Chem. 45, 2139-2149.

Vedani, A. and Huhta, D. W. (1991). An algorithm for the systematic solvation of proteins based on the directionality of hydrogen bonds. J. Am. Chem. Soc. 113, 5860-5862.

Vedani, A. and Huhta, D. W. (1990). A new force field for modeling metalloproteins. J. Am. Chem. Soc. 112, 4759-4767.

Vingtdeux, V., Dreses-Werringloer, U., Zhao, H. et al. (2008). Therapeutic potential of resveratrol in Alzheimer's diseases. BMC Neurosci. 9 (Suppl. 2), 6.

White paper on the strategy for a future chemicals policy (2003). Cf. http://europa.eu.int/ comm/environment/chemicals/whitepaper.htm

\section{Acknowledgements}

This research had been made possible by grants of the Swiss National Science Foundation, the Margaret and Francis-Fleitmann Foundation, Lucerne/Switzerland, the Doerenkamp-Zbinden Foundation, Zürich/Switzerland, the Jacques en Dolly Gazan Foundation, Zug/Switzerland, and the Foundation Animalfree Research, Zürich/Switzerland, which are all gratefully acknowledged.

\section{Correspondence to}

Prof. Dr. Angelo Vedani

Biographics Laboratory 3R

Friedensgasse 35

4056 Basel

Switzerland

e-mail: admin@biograf.ch 